\title{
Comparison of the effects of wave-particle interactions and the kinetic suprathermal electron population on the acceleration of the solar wind
}

\author{
S. W. Y. Tam and T. Chang \\ Center for Space Research, Massachusetts Institute of Technology, Cambridge, MA 02139, USA \\ Received 23 April 2002 / Accepted 5 September 2002

\begin{abstract}
Kinetic effects due to wave-particle interactions and suprathermal electrons have been suggested in the literature as possible solar wind acceleration mechanisms. Ion cyclotron resonant heating, in particular, has been associated with some qualitative features observed in the solar wind. In terms of solar wind acceleration, however, it is interesting to compare the kinetic effects of suprathermal electrons with those due to the wave-particle interactions. The combined effects of the two acceleration mechanisms on the fast solar wind have been studied by Tam \& Chang (1999a,b). In this study, we investigate the role of the suprathermal electron population in the acceleration of the solar wind. Our model follows the global kinetic evolution of the fast solar wind under the influence of ion cyclotron resonant heating, while taking into account Coulomb collisions, and the ambipolar electric field that is consistent with the particle distributions themselves. The kinetic effects due to the suprathermal electrons, which we define to be the tail of the electron distributions, can be included in the model as an option. By comparing the results with and without the inclusion of the suprathermal electron effects, we determine the relative importance of suprathermal electrons and wave-particle interactions in driving the solar wind. We find that although suprathermal electrons enhance the ambipolar electric potential in the solar wind considerably, their overall influence as an acceleration mechanism is relatively insignificant in a wave-driven solar wind.
\end{abstract}

Key words. Sun: solar wind - Sun: general - Sun: particle emission

\section{Introduction}

Since the pioneering work by Belcher \& Davis (1971), the existence of waves has been well established in the research of the fast solar wind. Wave-particle interactions have been considered as a possible solar wind heating mechanism, and their effects have been studied by a number of authors (e.g. Hollweg \& Turner 1978; Dusenbery \& Hollweg 1981; Marsch et al. 1982c; Isenberg 1984; Leer et al. 1992; Hu et al. 1997; Czechowski et al. 1998; Cranmer et al. 1999b; Hu \& Habbal 1999; Li et al. 1999; Tam \& Chang 1999a; Isenberg et al. 2000; Cranmer 2001; Gary et al. 2001; Liewer et al. 2001; Tam \& Chang 2001; Tu \& Marsch 2001; Vocks \& Marsch 2001). Experimentally, there has been increasing evidence suggesting that wave-particle interactions play a significant role in the evolution and acceleration of the solar wind. First, the proton velocity distributions observed by the Helios spacecraft featured an anisotropy in the core region, where the temperature in the perpendicular direction was higher than that in the direction parallel to the magnetic field (Marsch et al. 1982b). Such an

Send offprint requests to: S. W. Y. Tam, e-mail: wyt@space.mit.edu anisotropy was an indication of the presence of ion perpendicular heating in the solar wind. The observed proton velocity distributions were also frequently characterized by double peaks in the field-aligned direction. The formation of these double peaks has recently been associated with kinetic ion cyclotron resonances involving sunward propagating electromagnetic waves (Tam \& Chang 1999a,b).

The differential speeds between the alpha particles and the protons in the solar wind, which were perhaps the most unexpected experimental results observed by the Helios spacecraft, may also be highly related to wave-particle interactions. Measurements by the spacecraft revealed that the helium ions have a higher outflow speed compared with the protons (Marsch et al. 1982a), especially in the fast solar wind. Indeed, more recent observations by the WIND and the Ulysses spacecraft have also arrived at a similar conclusion (Steinberg et al. 1996; Feldman et al. 1996). Because the helium ions have a smaller charge-to-mass ratio compared with the protons, the observed ion differential speeds were inconsistent with the idea of the field-aligned ambipolar electric field being the main solar wind acceleration mechanism. A physical mechanism that has been demonstrated to preferentially accelerate helium ions 
rather than protons is kinetic ion resonant heating by electromagnetic waves in the ion gyrofrequency range (Tam \& Chang 1999a,b). These waves may be produced by the small-scale reconnections near the coronal region. Recently, Chang (2002) has suggested that such type of intermittent turbulence may be associated with the "complexity" generated by the sporadic localized mergings and interactions of the coherent magnetic structures near the coronal holes. Measurements of the power spectra of these waves by the Helios spacecraft indicated that the wave power decreases with frequency (Bavassano et al. 1982; Denskat \& Neubauer 1982). Note that the alpha particles have a lower gyrofrequency than the protons. Therefore, in general, more wave power is available to the helium ions than to the protons, at least in the observed regimes (0.3-1 AU). Extrapolating the power spectrum to distances close to the sun, Tam \& Chang (1999a) have shown that the kinetic waveparticle interactions in general transfer more energy to a helium ion than to a proton. Although the energy is mainly transferred into the transverse direction, it converts into the field-aligned direction downstream in the solar wind due to the mirror effect, leading to a preferential acceleration of the helium ions.

Experimental data from SOHO have provided even more evidence in support of the significance of wave-particle interactions in the fast solar wind. The observed $\mathrm{O}^{5+}$ ions in the extended corona deviate significantly from those of a thermal distribution. Their velocity distributions are strongly anisotropic, with $T_{\perp}>T_{\|}$, and perpendicular kinetic temperature higher than $10^{8} \mathrm{~K}$ (Kohl et al. 1998; Cranmer et al. 1998; 1999a). It has been pointed out by the investigators who analyzed the data that this evidence is consistent with ion cyclotron resonant heating.

Besides wave-particle interactions, other physical mechanisms have also been considered as possible drivers of the solar wind. The proposal of suprathermal electron effects has led to theory-data agreement in the ionospheric polar wind, which, like the solar wind, is an outflow of space plasmas along open magnetic field lines. The reason why suprathermal electrons may drive an outflowing plasma, such as the solar wind, is the following. Without taking wave-particle interactions into consideration, Scudder \& Olbert (1979) argued that due to the velocity-dependence of the Coulomb collisional depth and the global kinetic nature of the solar wind outflow, suprathermal tails could be formed in the electron distributions. To demonstrate this "velocity filtration effect", these authors used a simplified collisional operator to determine the evolution of an electron distribution along the solar wind. They were able to show that an electron distribution in the form of a Maxwellian at the corona could evolve into one with a notable tail population in the anti-sunward direction near 1 AU. Olbert (1982) further suggested that the heat flux contribution by the suprathermal electron tail may drive the solar wind. Such an idea has been applied to the ionospheric polar wind with photoelectrons playing the role of the suprathermal population (Tam et al. 1995; 1998), and has successfully addressed the various features observed by satellites. It has been shown that in the absence of wave-particle interactions, the suprathermal electron population can increase the ambipolar electric field, leading to higher ion outflow speeds in the polar wind. Because the ionospheric polar wind and the solar wind are analogous phenomena - both are outflow of space plasmas along open magnetic field lines - it is reasonable to expect that suprathermal electron effects may accelerate the ions in the solar wind.

The combined effects of ion cyclotron resonant heating and the suprathermal electron population have been considered in a solar wind study by Tam \& Chang (1999a). The study was the first to describe the global kinetic evolution and acceleration of the fast solar wind under the influence of the wave-particle interactions, Coulomb collisions, and an ambipolar electric field that was consistent with the particle distributions themselves. Results of the investigation associated the wave-particle interactions with some of the solar wind observations discussed earlier, including the differential speed between the helium ions and the protons, and the double-peaked proton velocity distributions. The bulk acceleration of the solar wind demonstrated in the study, however, consisted of contributions due to both the ion resonances and the suprathermal electron effects. It was therefore unclear to what extent each of the two acceleration mechanisms contributes to the driving of the solar wind. Our goal in this study is to compare the relative importance of the kinetic effects due to suprathermal electrons and ion cyclotron resonances, and to determine which of the acceleration mechanisms is mostly responsible for the driving of the solar wind.

This paper is structured as follows: in Sect. 2, we shall describe our model and the technique we use to take into account the global kinetic effects of wave-particle interactions, as well as those of the suprathermal electron population. The influence on the solar wind due to these two physical mechanisms will be compared in Sect. 3. The comparison will enable us to determine the contribution by the velocity filtration effect in driving the solar wind. In Sect. 4, we shall consider the kinetic effects of suprathermal electrons from a more general perspective, not only limited to the velocity filtration effect. Finally, important points discussed in this article will be summarized in Sect. 5 .

\section{Model}

Our model in this study is based on a self-consistent hybrid technique originally developed to describe the ionospheric polar wind (Tam et al. 1995, 1998). Because the polar wind (as termed in analogy with the solar wind (Axford 1968; Banks \& Holzer 1968) is also an example of plasma outflow along open magnetic field lines, our present model retains the general features of the self-consistent hybrid model: it is based on an iterative scheme between fluid and kinetic calculations. In the fluid calculations, a set of equations determines the ambipolar electric field and the properties of the bulk thermal electrons, whose distributions are assumed to be in a drifting Maxwellian. To solve the fluid equations, we impose the quasi-neutrality and current-free constraints for the solar wind, and make use of the results from the kinetic calculations in the model. The kinetic part of the model consists of multiple components, each describing the evolution of a particle component along the solar wind. In the basic version of the model, the kinetic approach is applied only to two particle components: proton and alpha particle, the two major ion species in the solar wind. 
The distributions of these ions evolve under the influence of Coulomb collisions, an ambipolar electric field, and waveparticle interactions. Results based on the global evolution of these distributions are coupled into the fluid calculations. This coupling ensures the consistency of the ambipolar electric field with the particle distributions when an iteration between the fluid and kinetic parts of the model converges. In addition, a convergence of the results enables our kinetic calculations to correctly take into account the Coulomb collisions for the ions, including those among the same ion species.

Effects of kinetic ion resonant heating are incorporated into the model with a Monte Carlo technique. This technique was originally developed to model ion resonant heating in the auroral region, and has successfully addressed the existence of ion conics (Retterer et al. 1983). By including this technique into the model, we are able to incorporate the resonant effect of a given wave spectrum into an otherwise self-consistent solar wind description.

To be more quantitative on the description of our kinetic calculations, we solve the following steady-state collisional kinetic equations for the protons and the helium ions, the particle components that resonate with the waves:

$$
\begin{aligned}
{\left[v_{\|} \frac{\partial}{\partial s}\right.} & \left.-\left(g-\frac{q}{m} E_{\|}\right) \frac{\partial}{\partial v_{\|}}-v_{\perp}^{2} \frac{B^{\prime}}{2 B}\left(\frac{\partial}{\partial v_{\|}}-\frac{v_{\|}}{v_{\perp}} \frac{\partial}{\partial v_{\perp}}\right)\right] f_{j} \\
& =C_{j} f_{j}+D_{j} f_{j},
\end{aligned}
$$

where $s$ is the distance along the radial magnetic field line, $v_{\|}$ and $v_{\perp}$ denote velocities in the spacecraft frame, $f_{j}\left(s, v_{\|}, v_{\perp}\right)$ is the distribution function for the particle species $j, q$ and $m$ are the electric charge and mass of the species respectively, $E_{\|}$ is the field-aligned ambipolar electric field, $g$ is the gravitational acceleration, $B$ is the magnetic field, $B^{\prime} \equiv \mathrm{d} B / \mathrm{d} s, C_{j}$ is a Coulomb collisional operator for the species $j$, and

$D_{j}=\frac{\partial}{\partial v_{\|}} D_{j \|} \frac{\partial}{\partial v_{\|}}+\frac{1}{v_{\perp}} \frac{\partial}{\partial v_{\perp}}\left(v_{\perp} D_{j \perp} \frac{\partial}{\partial v_{\perp}}\right)$

is an operator that describes resonant heating, with $D_{j \|}$ and $D_{j \perp}$ being quasilinear diffusion coefficients that represent heating in the parallel and perpendicular directions respectively. The expressions for $D_{j \|}$ and $D_{j \perp}$, in general, depend on a number of factors, including the kind of wave-particle interactions, the conditions for resonances, the power of the waves, etc. Below, we discuss in detail the conditions that determine the diffusion coefficients in the study.

\subsection{Ion cyclotron resonant heating}

The wave-particle interactions to be described in Eqs. (1) and (2) are kinetic ion cyclotron resonances, which have been demonstrated to preferentially accelerate the helium ions over the protons (Tam \& Chang 1999a,b). The resonances that we consider involve electromagnetic waves whose wavevector component $k_{\perp} \ll k_{\|} \approx k$. Because the thermal speeds of the ions are much smaller than the local Alfvén speed near the corona, where the majority of the resonant heating occurs, the properties of the waves can be approximated by a cold plasma dispersion relation. Moreover, due to the relatively low thermal speeds, few ions are able to resonate with right-hand polarized waves. Therefore, the only waves that we consider in this study are left-hand polarized, and are in the Alfvén/ion cyclotron branch, which in fact consists of two sub-branches for the two-ion (proton and helium) plasma in our model. In the solar wind frame, the lower sub-branch covers all frequencies up to the helium gyrofrequency, $\Omega_{\alpha}$. The upper sub-branch plateaus at the proton gyrofrequency $\Omega_{\mathrm{p}}$ for large $k$, and its frequency limit at infinite wavelength is above $\Omega_{\alpha}$, leaving a gap between the two sub-branches. For an ion (proton or alpha particle) with parallel velocity $v^{\prime} \|$ in the solar wind frame to resonate with the left-hand polarized waves, the following condition has to be satisfied:

$\omega^{\prime}-k v^{\prime} \|=\Omega$

where $\omega^{\prime}$ is the wave frequency in the solar wind frame, and $\Omega$ is the gyrofrequency of the ion. The wave frequency observed by the spacecraft is Doppler-shifted to $\omega$, given by:

$\omega=\omega^{\prime}+k U$

where $U$ is the local solar wind speed. Note that the sign of $k$ can be positive or negative, representing outward (antisunward) or inward (sunward) propagating waves respectively.

Although the waves in the solar wind propagate predominantly outward, a statistical analysis of the power spectra measured by the Helios spacecraft indicated that inward propagating waves constitute a considerable amount of power in both the fast and slow solar wind (Tu \& Marsch 1990). The study showed that in the fast solar wind, the power of inward propagating waves $\left(e_{-}\right)$tends to approach its outward counterpart $\left(e_{+}\right)$as the radial distance increases. The results of the study motivated us to include inward propagating waves in our model. We assume the following relation for the wave power ratio in the interplanetary space:

$e_{-} / e_{+}=1-\mathrm{e}^{-(s / 3)^{2}}$,

where $s$ is the heliocentric distance in AU. Note that with Eq. (5), we assume that the inward propagating waves constitute only $2.4 \times 10^{-6}$ of the total wave power at $1 R_{\odot}$ (solar radius). Even with such a small ratio of $e_{-} / e_{+}$near the corona, the presence of these sunward propagating waves have been shown to lead to double-peaked proton velocity distributions in the solar wind (Tam \& Chang 1999a). The reason for the formation of the non-thermal feature is as follows. Because $\omega^{\prime}<\Omega_{\mathrm{p}}$ for all the waves in the ion cyclotron branch, it is clear from Eq. (3) that protons with $v^{\prime} \|<0$ can only resonate with the outward propagating waves, while those with $v^{\prime} \|>0$ only interact with the incoming waves. Note that at any given distance, the antisunward and sunward propagating waves heat two distinct proton populations in the velocity space. If incoming waves were absent, pitch-angle diffusion of the protons due to the waveparticle interactions could only occur at velocity space where $v^{\prime} \|<0$. The presence of sunward propagating waves enables the proton distributions to undergo two distinct sets of pitchangle diffusion, one at $v^{\prime} \|<0$ and the other at $v^{\prime} \|>0$. As the solar wind evolves along the flow, each of these two sets 
of velocity-space diffusion gives rise to a hump in the proton distributions because of the effect of mirror folding, thereby forming the observed double-peaked feature. Later, we shall provide evidence to support the association between doublepeaked proton velocity distributions and sunward propagating waves when we briefly discuss the effect on the solar wind due to a different $e_{-} / e_{+}$ratio.

The power of the waves in the solar wind decreases with frequency, as indicated by the Helios measurements of the magnetic field spectra (Bavassano et al. 1982; Denskat \& Neubauer 1982). The dependence of the wave power on frequency can generally be described by a power law. It was also found that the wave power decreases with radial distance, and the dependence can also be described by a power law, at least in the observed regime (Bavassano et al. 1982). In our model, we assume a magnetic field power spectrum based on these experimental results. We extend the power-law relation to higher frequencies. As for the dependence on radial distance, we assume a power-law relation and interpolate to obtain the magnetic field power between the two experimental limits $(0.29$ and $0.87 \mathrm{AU})$. For radial distances outside the measurement range, we assume the wave power to be proportional to $s^{-4.2}$.

With the resonance condition and magnetic field power spectra defined, we are able to determine the frequencies and power of the waves that are in resonance with the ions. The two-ion (proton and helium) cold plasma dispersion relation for the left-hand polarized waves in the solar wind frame, which we consider to be the frame where both the combined ion populations and the total electron population carry zero current, can be expressed as:

$\left(k v_{\mathrm{A}}\right)^{2}=\omega^{\prime 2}\left[\frac{1-\omega^{\prime} / \omega_{0}}{\left(1-\omega^{\prime} / \Omega_{\alpha}\right)\left(1-\omega^{\prime} / \Omega_{\mathrm{p}}\right)}\right]$,

where $v_{\mathrm{A}}$ is the Alfvén speed, and

$\omega_{0}=\Omega_{\alpha}\left(n_{\mathrm{p}}+4 n_{\alpha}\right) /\left(n_{\mathrm{p}}+2 n_{\alpha}\right)$

is a frequency that depends on the densities of the protons $\left(n_{\mathrm{p}}\right)$ and the helium ions $\left(n_{\alpha}\right)$. Note that $\omega_{0}$ represents the longwavelength limit for the upper ion cyclotron sub-branch. One can see that Eq. (6) does not have any solution for $\Omega_{\alpha}<$ $\omega^{\prime}<\omega_{0}$, resulting in a frequency gap between the two ion cyclotron sub-branches. However, in the limit of a one-ion plasma $\left(n_{\alpha} \rightarrow 0\right)$, the frequency gap disappears (as $\omega_{0} \rightarrow \Omega_{\alpha}$ in Eq. (7)), and the dispersion relation reduces to a single branch. Equation (6), together with Eq. (3), determine the wavevectors and frequencies of the waves that resonate with the individual ions in the solar wind frame. These resonant frequencies are then Doppler-shifted to the spacecraft frame according to Eq. (4). The total wave power at these frequencies are then determined based on the interpolation/extrapolation scheme of the magnetic field spectrum described earlier.

The diffusion coefficients in Eq. (2) involve all of the considerations discussed above. Standard expressions for these coefficients can be found in, for example, Swanson (1989). But by taking into account all the considerations above, we arrive at a modified version of the diffusion coefficients:

$D_{\|}=\eta \frac{q^{2}}{4 m^{2}} \int \mathrm{d} \omega v_{\perp}^{2} P_{\mathrm{B}}(\omega / 2 \pi) \delta\left(\omega-k v_{\|}-\Omega\right)$,
$D_{\perp}=\eta \frac{q^{2}}{4 m^{2}} \int \mathrm{d} \omega(\Omega / k)^{2} P_{\mathrm{B}}(\omega / 2 \pi) \delta\left(\omega-k v_{\|}-\Omega\right)$,

where the $\delta$-functions reflect our emphasis on the resonance condition equivalent to Eqs. (3), $\omega$ and $k$ are related through Eqs. (3), (4) and (6), $P_{\mathrm{B}}$ is a function for the magnetic field wave power, which is based on our interpolation/extrapolation scheme of the Helios measurements, and depends on the sign of $k$ (direction of the wave propagation) as described earlier, and lastly,

$\eta=\eta_{0} \exp [(1-r) / 0.5]$

is an efficiency factor that adjusts the available wave power, and depends on the heliocentric distance $r$ (in the unit of $R_{\odot}$ ). The adjustment of the wave power is mainly for two reasons. First, the factor $\eta_{0}$ in Eq. (10) accounts for the fact that the left-hand circularly polarized waves constitute only a fraction of the total observed wave power. Second, the remaining factor on the right-hand side of the equation approximates the dissipation of the waves as they propagate along the magnetic field line from the sun. It is chosen based on the study by Cranmer et al. (1999b), who suggested that most of the waves originating from the solar surface are dissipated before reaching $1.5 R_{\odot}$.

\subsection{Kinetic suprathermal electron effects}

Up to this point, we have described the basic constituents of our model. This model enables us to follow the global evolution of the ion distributions along the solar wind flow, while taking into account the influence due to kinetic ion cyclotron resonant heating, Coulomb collisions, and an ambipolar electric field that is consistent with the particle distributions themselves. The kinetic effects of the ions are also incorporated into the overall solar wind picture through the coupling of their results with the fluid calculations. In fact, our modeling technique can readily incorporate the kinetic effects of another particle component. To do so, we simply add another kinetic component into the model to describe the particle population, and modify the quasi-neutrality and current-free conditions, as well as the fluid equations accordingly. This flexibility makes our model an ideal tool to investigate the global kinetic effects of suprathermal electrons on the solar wind.

To take into account the global kinetic effects of the suprathermal electrons, we solve a steady-state collisional kinetic equation for the population that comprises the tail portion of the electron Maxwellian distribution at the lower boundary of our model. The equation is similar to Eq. (1), but without the term that represents wave-particle interactions. In addition, the operator that describes the Coulomb collisions for the suprathermal electrons is simplified. The collisional operator in Eq. (1), such as the Fokker-Planck collisional operator, should have the following expression in its complete form (see, e.g., Ichimaru 1973; Book 1989):

$C_{j} f_{j}(\boldsymbol{v})=-\sum_{b} \nabla_{\boldsymbol{v}} \cdot \boldsymbol{J}_{j b}$, 
where the subscript $b$ represents the background species with which the particles of species $j$ interact, and

$$
\begin{gathered}
\boldsymbol{J}_{j b}(\boldsymbol{v})=2 \pi \Lambda_{j b} \frac{q^{2} q_{b}^{2}}{m} \int \mathrm{d}^{3} v^{\prime} \frac{\left(\boldsymbol{v}^{\prime}-\boldsymbol{v}\right)^{2} \boldsymbol{I}-\left(\boldsymbol{v}^{\prime}-\boldsymbol{v}\right)\left(\boldsymbol{v}^{\prime}-\boldsymbol{v}\right)}{\left|\boldsymbol{v}^{\prime}-\boldsymbol{v}\right|^{3}} \\
\cdot\left[\frac{1}{m_{b}} f_{j} \nabla_{\boldsymbol{v}^{\prime}} f_{b}\left(\boldsymbol{v}^{\prime}\right)-\frac{1}{m} f_{b}\left(\boldsymbol{v}^{\prime}\right) \nabla_{\boldsymbol{v}} f_{j}\right],
\end{gathered}
$$

where $\Lambda_{j b}$ is the Coulomb logarithm, and $\boldsymbol{I}$ is the unit dyad. In the full collisional description, such as that for the ions, the background includes the thermal electrons and the two major ion species. But for the suprathermal electrons, we take into account their Coulomb interaction only with the thermal electrons. The interaction among the suprathermal electrons themselves are not considered due to their low relative density. With these approximations, and the assumption that the background thermal electron distribution is in the form of a drifting Maxwellian,

$f_{\mathrm{e}}(\boldsymbol{v})=n_{\mathrm{e}}\left(\frac{m_{\mathrm{e}}}{2 \pi T_{\mathrm{e}}}\right)^{3 / 2} \exp \left[-\frac{m_{\mathrm{e}}\left(\boldsymbol{v}-\boldsymbol{u}_{\mathrm{e}}\right)^{2}}{2 T_{\mathrm{e}}}\right]$,

the collisional term in the kinetic equation for the suprathermal electrons becomes:

$C_{\mathrm{s}} f_{\mathrm{s}}=-\nabla_{\boldsymbol{v}} \cdot\left(\boldsymbol{a}_{\mathrm{colll}, s} f_{\mathrm{s}}\right)+\nabla_{\boldsymbol{v}} \cdot\left(\boldsymbol{D}_{\mathrm{coll}, s} \cdot \nabla_{\boldsymbol{v}} f_{\mathrm{s}}\right)$,

where

$\boldsymbol{a}_{\mathrm{coll}, s}=-\left(m_{\mathrm{s}} / m_{\mathrm{e}}\right) v_{0} \boldsymbol{c} \Psi\left(x_{\mathrm{e}}\right)$,

$$
\begin{aligned}
\boldsymbol{D}_{\text {coll }, \boldsymbol{s}}= & \frac{\gamma_{0}}{2} \boldsymbol{c} \boldsymbol{c} \frac{\Psi\left(x_{\mathrm{e}}\right)}{x_{\mathrm{e}}} \\
& +\frac{v_{0}}{2}\left(c^{2} \boldsymbol{I}-\boldsymbol{c} \boldsymbol{c}\right)\left[\left(1-\frac{1}{2 x_{\mathrm{e}}}\right) \Psi\left(x_{\mathrm{e}}\right)+\Psi^{\prime}\left(x_{\mathrm{e}}\right)\right],
\end{aligned}
$$

with

$\boldsymbol{c}=\boldsymbol{v}-\boldsymbol{u}_{\mathrm{e}}$,

$v_{0}=4 \pi \Lambda_{\mathrm{se}} n_{\mathrm{e}} q_{\mathrm{s}}^{2} q_{\mathrm{e}}^{2} / m_{\mathrm{s}}^{2} c^{3}$,

$x_{\mathrm{e}} \equiv m_{\mathrm{e}} c^{2} / 2 T_{\mathrm{e}}$,

$\Psi(x)=\frac{2}{\sqrt{\pi}} \int_{0}^{x} \mathrm{~d} t \sqrt{t} \mathrm{e}^{-t}$,

$\Psi^{\prime}(x) \equiv \mathrm{d} \Psi / \mathrm{d} x$, and the subscripts "s" and "e" stand for the suprathermal and thermal electrons respectively (thus, $q_{\mathrm{s}}=q_{\mathrm{e}}$ and $m_{\mathrm{s}}=m_{\mathrm{e}}$ ). Equation (14) indicates that the Coulomb collisions have two effects on the suprathermal electrons: to provide a deceleration and a diffusion. The expression for $\boldsymbol{a}_{\mathrm{coll}, \mathrm{s}}$ in Eq. (15) suggests that the collisions act as a friction between the thermal electrons and the individual suprathermal electrons. The Coulomb interaction also leads to a diffusion of the suprathermal electrons in the velocity space, characterized by the tensor $\boldsymbol{D}_{\text {coll,s.s. }}$. Note that mathematically, $\boldsymbol{D}_{\text {coll,s }}$ plays a similar role as $D_{j \|}$ and $D_{j \perp}$ of Eqs. (2) in the governing kinetic equation. Therefore, the Monte Carlo technique in our model can also describe the diffusion due to the Coulomb collisions. According to Eqs. (15) and (16), both the diffusion and the friction are characterized by a collisional frequency $v_{0}$. Because $v_{0}$ is proportional to $1 / c^{3}$ (see Eq. (18)), the more energetic suprathermal electrons are less collisional. Because of this relationship, Coulomb collisions are expected to enhance the tail portion of the total electron distribution as the particles travel downstream along the solar wind flow. In our model, the electrons at the tail portion of the distribution have to satisfy two criteria at $1 R_{\odot}$, the lower boundary in the calculations, in order to be considered suprathermal: $(1 / 2) m_{\mathrm{e}} v^{2}>8 T_{\mathrm{e} 0}$ and $v_{\|}>0$, where $T_{\mathrm{e} 0}$ is the thermal electron temperature at $1 R_{\odot}$.

With the kinetic effects of suprathermal electrons being considered, the total electron distributions can no longer be in the form of a drifting Maxwellian. The distributions are now formed by the combination of a drifting Maxwellian for the thermal electrons, and the results from the kinetic calculations for the suprathermal electrons. As in the basic version of the model, the various parameters that constitute the drifting Maxwellian distributions for the thermal electrons - as well as the ambipolar electric field - are determined in the fluid calculations.

The density and drift velocity of the thermal electrons are determined from the quasi-neutrality and current-free constraints that characterize the solar wind, with input of densities and flow velocities of the other particle components (proton, helium, and suprathermal electron) obtained from the kinetic part of the model. In particular, the current-free condition is:

$n_{\mathrm{e}} u_{\mathrm{e}}+n_{\mathrm{s}} u_{\mathrm{s}}=n_{\mathrm{p}} u_{\mathrm{p}}+2 n_{\alpha} u_{\alpha}$,

where the subscripts "p" and $\alpha$ stand for the protons and the alpha particles respectively. The density and drift velocity profiles of the thermal electrons (and in fact, of every plasma component) should also satisfy the continuity equation:

$\frac{\partial}{\partial s}\left(\frac{n u}{B}\right)=0$

However, note that both Eqs. (21) and (22) involve the number flux of the particle species. Because the profiles of the particle components obtained from the kinetic part of the model have already satisfied Eq. (22), the imposition of the currentfree constraint throughout the whole simulation range renders thermal electron density and velocity profiles that would also satisfy Eq. (22).

The thermal electron temperature, together with the ambipolar electric field, are obtained from the moment equations of the next two higher orders, i.e. the momentum and energy transfer equations for the whole electron population:

$$
\begin{aligned}
B \frac{\partial}{\partial s}( & \left.\frac{n_{\mathrm{e}} T_{\mathrm{e}}+n_{\mathrm{e}} m_{\mathrm{e}} u_{\mathrm{e}}^{2}+n_{\mathrm{s}} T_{\mathrm{s} \|}+n_{\mathrm{s}} m_{\mathrm{e}} u_{\mathrm{s}}^{2}}{B}\right) \\
& +\left(n_{\mathrm{e}}+n_{\mathrm{s}}\right)\left(m_{\mathrm{e}} \frac{\partial \Phi_{G}}{\partial s}-e \frac{\partial \Phi_{E}}{\partial s}\right) \\
& +\frac{B^{\prime}}{B}\left(n_{\mathrm{e}} T_{\mathrm{e}}+n_{\mathrm{s}} T_{\mathrm{s} \perp}\right)=\frac{\delta M_{\mathrm{e}}}{\delta t}+\frac{\delta M_{\mathrm{s}}}{\delta t}
\end{aligned}
$$




$$
\begin{aligned}
B \frac{\partial}{\partial s}\left[\frac{n_{\mathrm{e}} u_{\mathrm{e}}}{B}\left(\frac{5}{2} T_{\mathrm{e}}+\frac{m_{\mathrm{e}} u_{\mathrm{e}}^{2}}{2}\right)+\frac{Q_{\mathrm{w}, \mathrm{s}}}{B}\right. \\
\left.+\left(\frac{n_{\mathrm{e}} u_{\mathrm{e}}}{B}+\frac{n_{\mathrm{s}} u_{\mathrm{s}}}{B}\right)\left(m_{\mathrm{e}} \Phi_{\mathrm{G}}-e \Phi_{\mathrm{E}}\right)\right] \\
=\frac{\delta E_{\mathrm{e}}}{\delta t}+\frac{\delta E_{\mathrm{s}}}{\delta t}
\end{aligned}
$$

where $e$ is the elementary charge, $\Phi_{\mathrm{G}}$ is the gravitational potential, $\Phi_{\mathrm{E}}$ is the potential for the field-aligned ambipolar electric field, $Q_{\mathrm{w}, \mathrm{s}}$ is the energy flux of the suprathermal electron population, obtained from the kinetic calculations, and $\delta M_{\mathrm{e}, \mathrm{s}} / \delta t$ and $\delta E_{\mathrm{e}, \mathrm{s}} / \delta t$ represent the rates of momentum and energy transfers to the thermal and suprathermal electrons due to Coulomb collisions. Unlike the standard moment method (Schunk 1977), where the collisional transfer values are completely based on assumed distribution functions, such as a Maxwellian, the rates of momentum and energy transfers in Eqs. (23) and (24) are determined based on the results of the kinetic components of our model. By calculating the momentum and energy transferred to the ions from the electrons in our kinetic simulations, we find these collisional transfer values with momentum and energy conservation, which results from the anti-symmetries of the Coulomb collisional operator in Eq. (12). However, we should note that the suprathermal electrons are not included in the collisional background for the ions. Nor are the ions included in the collisional background for the suprathermal electrons. These collisional values, therefore, do not take into account the transfer between the ions and the suprathermal electrons. Fichtner et al. (1996) studied the collisional transfers based on a number of distribution functions. Their results indicated that the transfer values for some non-Maxwellian distributions could be significantly different from those for a Maxwellian. However, they also noted that for distributions whose core regions do not deviate significantly from a Maxwellian, their collisional transfer values are similar to the Maxwellian case. As we shall show, the suprathermal electrons in our calculations do not significantly affect the core of the total electron distributions. Therefore, we believe that the transfer values due to collisions between the suprathermal electrons and the ions are negligible.

We note that the formulation of the fluid calculations is simpler when the kinetic effects of suprathermal electrons are not considered. In that case, the total electron distributions consist only of the thermal electrons, and, by assumption, are in the form of a drifting Maxwellian. One may then drop all the terms with subscript "s" in Eqs. (21), (23) and (24) to recover the fluid equations. However, whether suprathermal electron effects are considered or not, when the results of both the fluid and kinetic parts of our model converge, not only the collisional operators in the kinetic calculations provide a self-consistent background for the Coulomb interactions of the ions, but the ambipolar electric field is also consistent with the particle distributions themselves. Convergence of the results thus renders physically meaningful solutions for our solar wind model.

\section{Comparison of solar wind driving mechanisms}

\subsection{Strong wave-driven case}

As discussed, we can incorporate the suprathermal electron effects into our solar wind model by including the optional kinetic component described in Sect. 2.2. Because interactions between the electrons and the waves are not considered, the kinetic suprathermal electron effects in our calculations are essentially the velocity filtration effect proposed by Scudder \& Olbert (1979). Thus, by comparing solutions generated with and without the optional suprathermal electron component being included, we can identify the contribution by the velocity filtration effect in driving the solar wind.

We have generated two such solar wind solutions for the range between $1 R_{\odot}$ and $1 \mathrm{AU}$ with identical parameters and boundary conditions. The interplanetary magnetic field that we use is based on a dipole + quadrupole + current sheet model (Banaszkiewicz et al. 1998):

$$
\begin{aligned}
B(r)=2.21\left[\frac{2}{\left(r / R_{\odot}\right)^{3}}+\frac{4.5}{\left(r / R_{\odot}\right)^{5}}\right. & \\
& \left.+\frac{1}{1.538\left(r / R_{\odot}+1.538\right)^{2}}\right] \text { Gauss. }
\end{aligned}
$$

Boundary and initial conditions are imposed at the lower end of the calculations: a thermal electron temperature of $100 \mathrm{eV}$ is assumed. Initial velocity distributions for the protons and helium ions are the upper halves of Maxwellian distributions, whose temperatures are also $100 \mathrm{eV}$. The proton and helium ion densities are respectively $4.2 \times 10^{7}$ and $4.0 \times 10^{6} \mathrm{~cm}^{-3}$. The parameter $\eta_{0}$ that characterizes the strength of the available wave power is 0.016 . As we shall discuss below, such a value for $\eta_{0}$ corresponds to the case of a strong wave-driven solar wind.

Comparing the two solar wind solutions, we find that the most noticeably different results are the shape of the total electron distributions. Although in both cases we begin with a total electron distribution in the form of a Maxwellian at $1 R_{\odot}$, we find that the distributions become different downstream in the flow. Figure 1 shows the total electron distributions at two different locations. At $0.1 \mathrm{AU}$, in the solution where global kinetic effects are considered for the suprathermal electrons, a significant tail in the outward portion of the total electron distribution has already been developed. Downstream in the solar wind, even though the temperature of the thermal electrons is reduced, the tail remains as strong (middle panel of Fig. 1). Thus, relative to the thermal core, the tail portion of the electron distribution is more enhanced downstream. The formation and enhancement of this non-thermal feature verify Scudder \& Olbert's (1979) idea of the velocity filtration effect.

The deviation of the total electron distribution from the Maxwellian approximation can be seen more clearly in the bottom panel of Fig. 1, where the reduced distributions in the parallel direction for the two solutions are shown. It is evident that while the suprathermal electrons have a very significant contribution in the tail portion of the distribution, they do not really affect the core region. In fact, by comparing the overall electron temperature in the two solutions, we find that the suprathermal electrons do not affect the results by a significant 

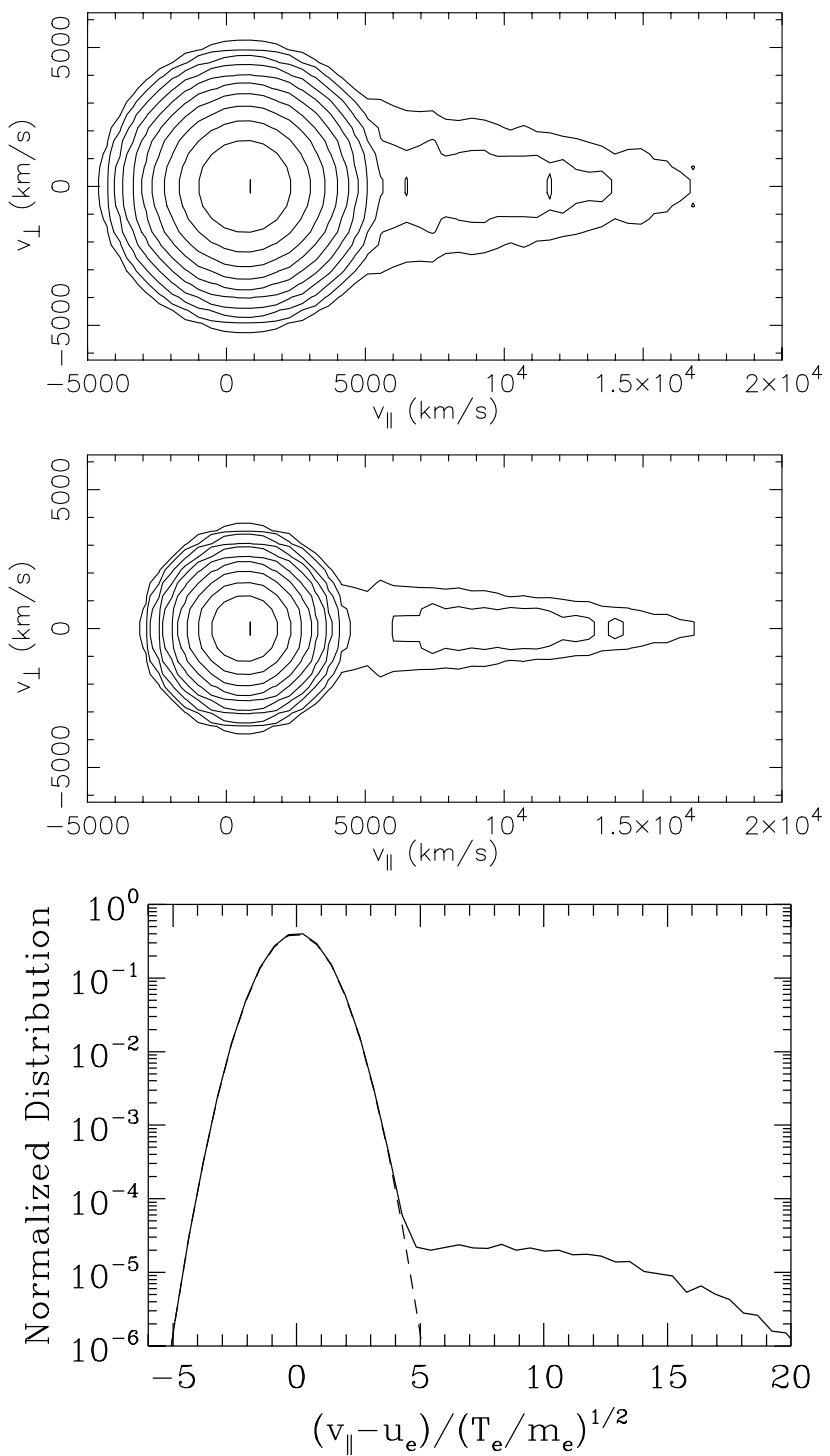

Fig. 1. Top and middle panels: contour plots for the total electron distributions at $0.1 \mathrm{AU}$ (top) and $0.4 \mathrm{AU}$ (middle) for the case that includes suprathermal electrons. Bottom panel: reduced electron distributions in the parallel direction at $0.4 \mathrm{AU}$. Solid line is the distribution for the case where global kinetic effects of the suprathermal electrons are included. Dashed line is the result with a Maxwellian approximation for the entire electron population.

amount (Fig. 2). Therefore, our test-particle approximation for the suprathermal electrons regarding their Coulomb collisions seems reasonable.

The formation of the suprathermal tail in the electron distributions leads to an outward total electron heat flux throughout the solar wind, as opposed to a zero heat flux with a Maxwellian approximation. As shown in Fig. 3, the profile of this outward electron heat flux essentially follows a power law from beyond $10 R_{\odot}(\approx 0.05 \mathrm{AU})$. Although the heat flux profile has a powerlaw index of -2.06 , which seems to agree with the estimates based on a number of fast solar wind events observed by the Helios spacecraft (Pilipp et al. 1990), its value at $1 \mathrm{AU}$, like the electron temperature, is about an order of magnitude too low compared with the observations (see Figs. 2 and 3). However,

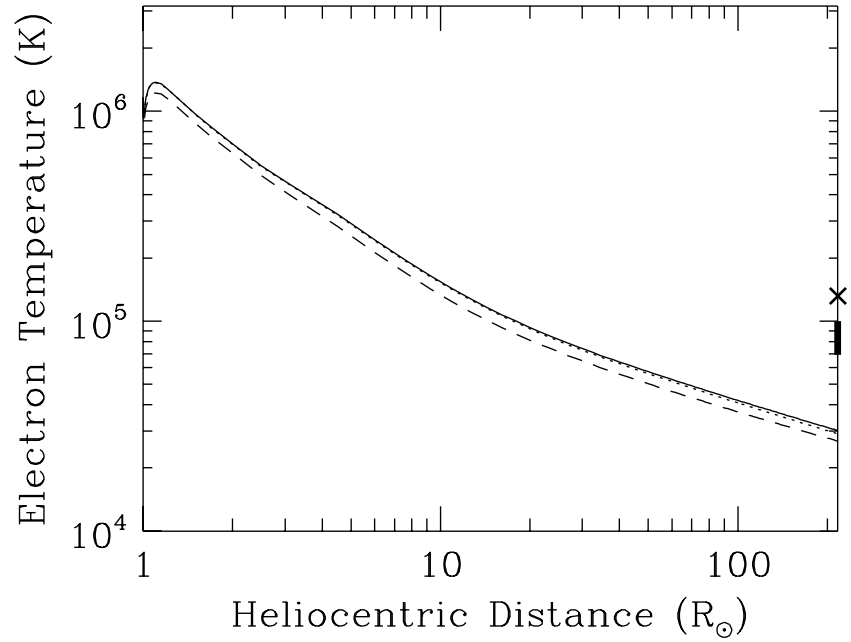

Fig. 2. Solid (dotted) line: parallel (perpendicular) temperature of the total electron population for the case where global kinetic effects of the suprathermal electrons are included. Dashed line: electron temperature for the Maxwellian approximation. The thick line segment and the cross $(x)$ at the right of the plot correspond to the core electron temperatures observed by the Helios (Pilipp et al. 1990) and Ulysses (Scime et al. 1994) spacecraft respectively.

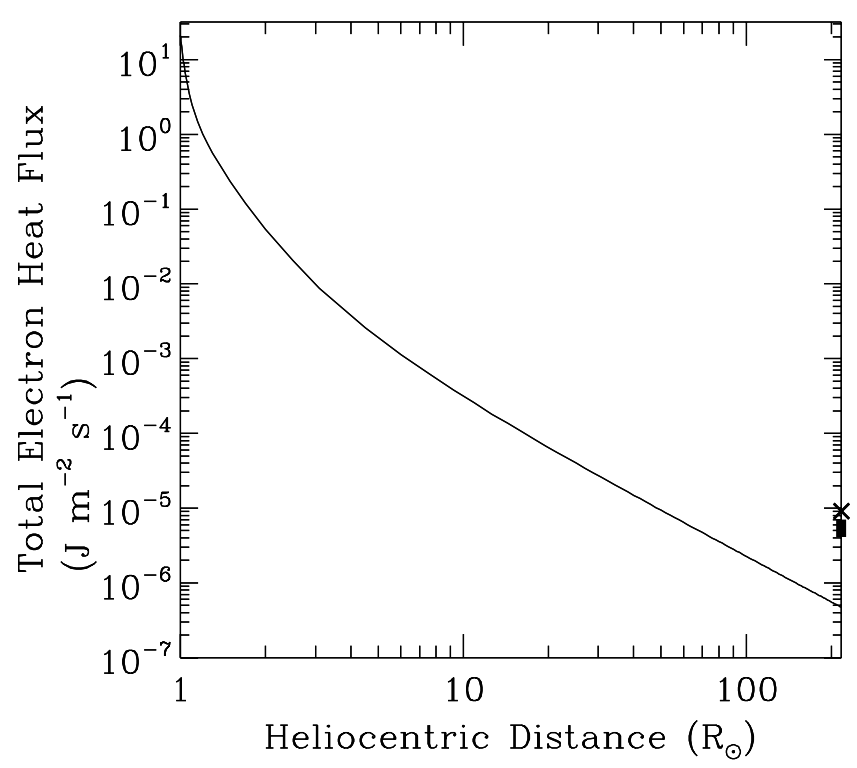

Fig. 3. Profile of the total electron heat flux from $1 R_{\odot}$ to $1 \mathrm{AU}$ in the case where the kinetic effects of the suprathermal electrons are included. The meaning of the thick line segment and the cross $(\mathbf{x})$ is similar to that in Fig. 2.

it has been shown that kinetic wave-particle interactions involving the solar wind electrons can increase the temperature and the heat flux of the species remarkably (Tam \& Chang 2001). In the present study, we exclude the electron heating so as to present a clearer picture of the impact by the velocity filtration effect.

The suprathermal electron population influences the ion species mainly through the ambipolar electric field. The ambipolar electric potential profiles for the two solar wind solutions are shown in Fig. 4. With a Maxwellian approximation for the entire electron population, the overall electric potential 


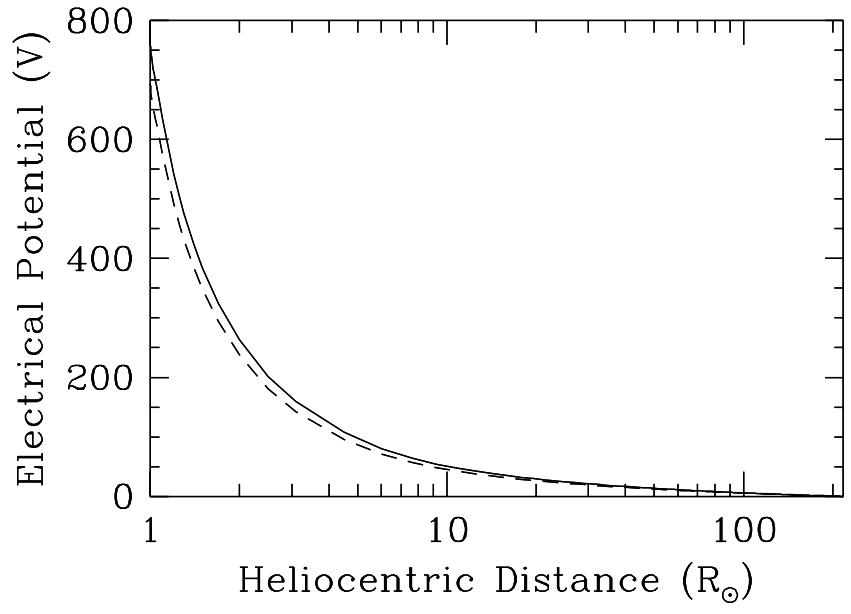

Fig. 4. Ambipolar electric potential profiles. Solid line: kinetic suprathermal electron effects included; dashed line: with a Maxwellian approximation for the entire electron distribution.

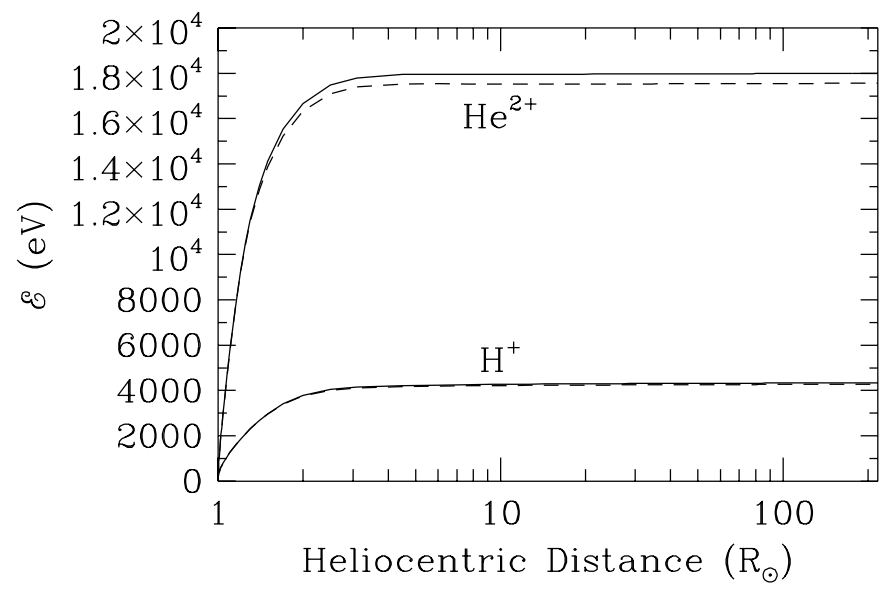

Fig. 5. $\mathcal{E}$ for protons and helium ions. Solid lines: kinetic suprathermal electron effects included; dashed lines: with a Maxwellian approximation for the entire electron distributions.

drop is about $700 \mathrm{~V}$ from $1 R_{\odot}$ to $1 \mathrm{AU}$. The potential difference increases by about $70 \mathrm{~V}$ when kinetic suprathermal electron effects are taken into account. Such an increase suggests that the velocity filtration effect can accelerate the ions in the solar wind.

To evaluate the significance of the velocity filtration effect as a solar wind driving mechanism, we shall compare its contribution with those from other physical mechanisms in terms of energy input to the ion populations. Besides the kinetic effect that associates with the suprathermal electrons, there are several physical processes affecting the ion energy in the solar wind. First, ion cyclotron resonant heating provides a direct source of energy input for both the protons and helium ions. In addition, Coulomb collisions allow energy exchange between different species in the solar wind. The energies of the ions are affected by these two physical processes not only directly, but also indirectly through the influence of these mechanisms on the ambipolar electric field. As the ions travel downstream along the solar wind flow, their kinetic energy increases due to the sunward gradient of the ambipolar electric potential (see Fig. 4), but at the same time, some of the kinetic energy is converted into gravitational potential energy. Whether an ion can

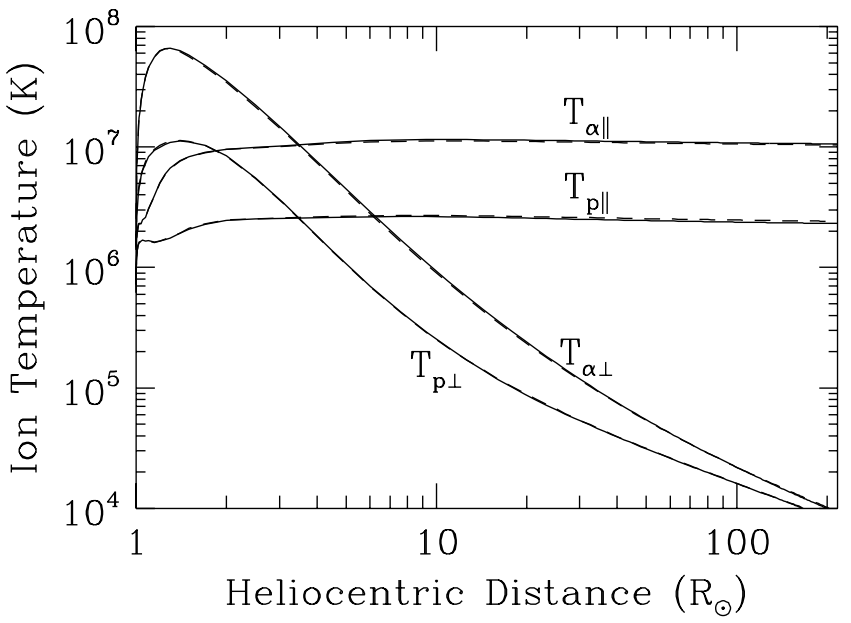

Fig. 6. Ion temperature profiles. Solid lines: kinetic suprathermal electron effects included; dashed lines: with a Maxwellian approximation for the entire electron distributions.

reach large radial distances in the solar wind depends whether the kinetic energy it gains is high enough to overcome the gravitational potential. Therefore, we evaluate the significance of a physical mechanism in driving the solar wind by comparing its contribution to the energy input for the ions with the contributions from all the physical processes combined. Because we are interested in comparing only the processes that may drive the solar wind, we shall exclude the gravitational force in our energy consideration. After all, the gravitational potential profile is invariant under all solar wind conditions, and is independent of the presence of other physical processes. Hence, we define the following quantity for each ion species for our purpose of energy comparison:

\section{$\mathcal{E} \equiv$ average kinetic energy \\ + gravitation potential energy per ion.}

For a given ion species in our model, its increase in $\mathcal{E}$ along the solar wind flow is due to a combination of energy input from the ambipolar electric field, wave-particle interactions, and Coulomb collisions with other species. In particular, the ambipolar electric field also reflects the influence due to waveparticle interactions, and Coulomb collisions, and, in the case where the kinetic suprathermal electron effects are included, the velocity filtration effect as well. The profiles of $\mathcal{E}$ for the protons and helium ions in our two solar wind solutions are shown in Fig. 5. It appears from the figure that the difference in $\mathcal{E}$ is very small between the two solutions. Indeed, we find that with the kinetic effects of the suprathermal electrons taken into account, the increase in $\mathcal{E}$ for the protons from $1 R_{\odot}$ to $1 \mathrm{AU}$ is only $1.5 \%$ larger than in the case of a Maxwellian approximation. For the helium ions, the corresponding difference is $2.4 \%$.

Because the quantity $\mathcal{E}$ characterizes the average kinetic energy, it is related to both the temperature and average outflow speed of the species. The temperature profiles of the ions in the two solar wind solutions are compared in Fig. 6. One can see from the figure that the influence on the proton and helium ion temperatures due to the velocity filtration effect is negligible. 


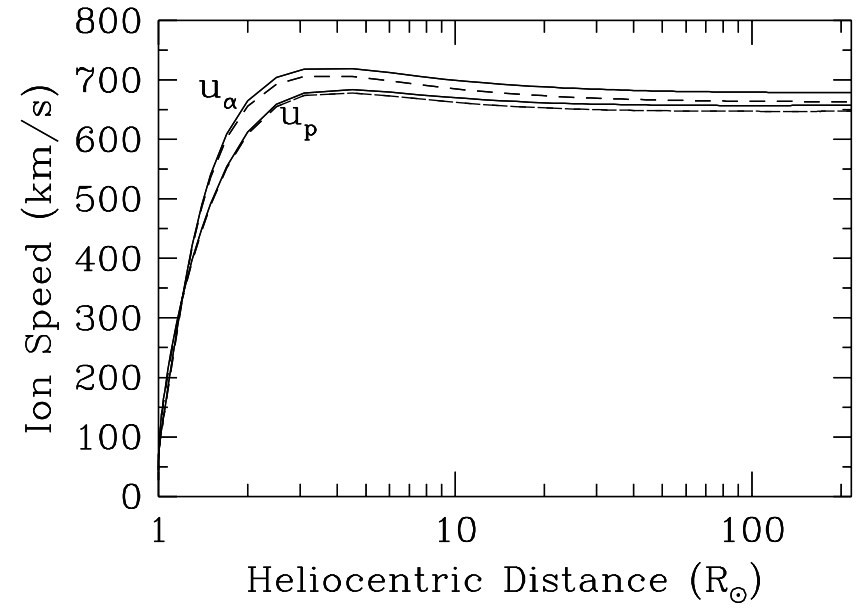

Fig. 7. Profiles of the proton and helium ion outflow speeds. Solid lines: kinetic suprathermal electron effects included; dashed lines: with a Maxwellian approximation for the entire electron distributions.

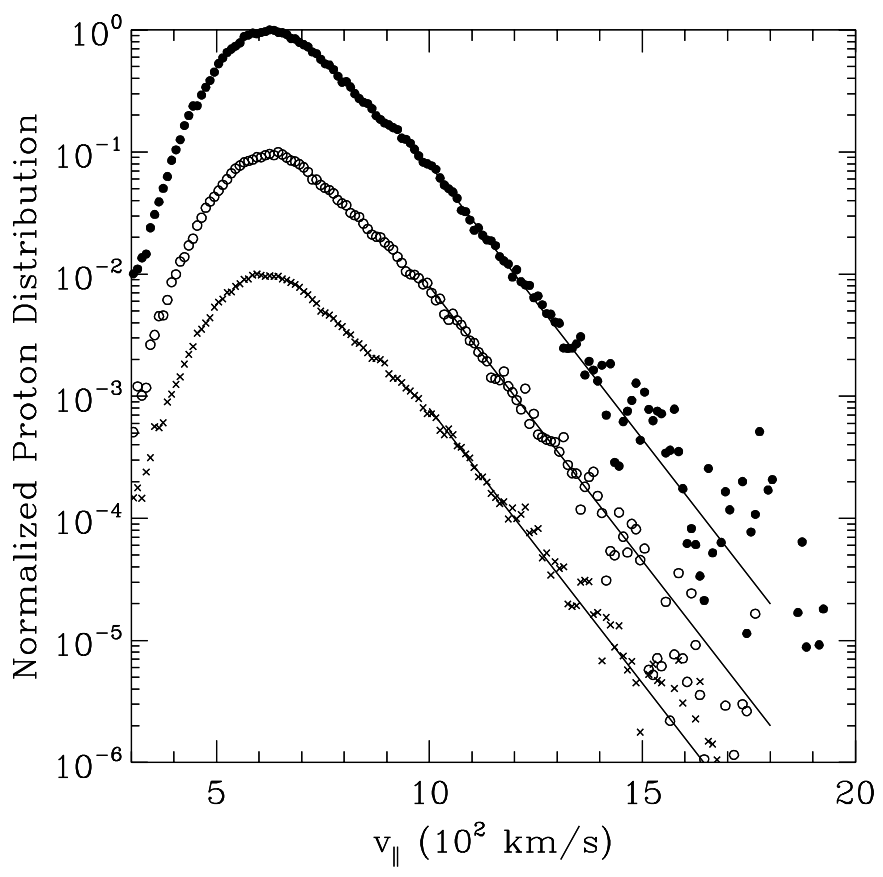

Fig. 8. Cuts of normalized proton velocity distributions at the $v_{\perp}=0$ plane at $0.2 \mathrm{AU}$. The crosses $(\times)$ corresponds to the solution based on a Maxwellian approximation for the total electron population. Open circles (o) are for the solution that includes kinetic suprathermal electron effects. The ratio $e_{-} / e_{+}$is given by Eq. (5) for both of these cases. Filled circles $(\bullet)$ are for $e_{-} / e_{+}$given by Eq. (26), with suprathermal electron effects considered. For clarity, the distributions are normalized differently so that their peak values are exactly one order of magnitude apart. The straight solid lines at the tail of the distributions are parallel and also exactly one order of magnitude apart.

The influence by the kinetic suprathermal electron effects on the ion outflow speeds can be seen in Fig. 7. The difference due to the velocity filtration effect is minimal, considering that the kinetic effect only lead to a $1.5 \%$ increase in the proton speed and a $2.3 \%$ increase in the helium ion speed at $1 \mathrm{AU}$. Note that even under a Maxwellian approximation for the overall electron distributions, the solar wind speed at $1 \mathrm{AU}$ is as

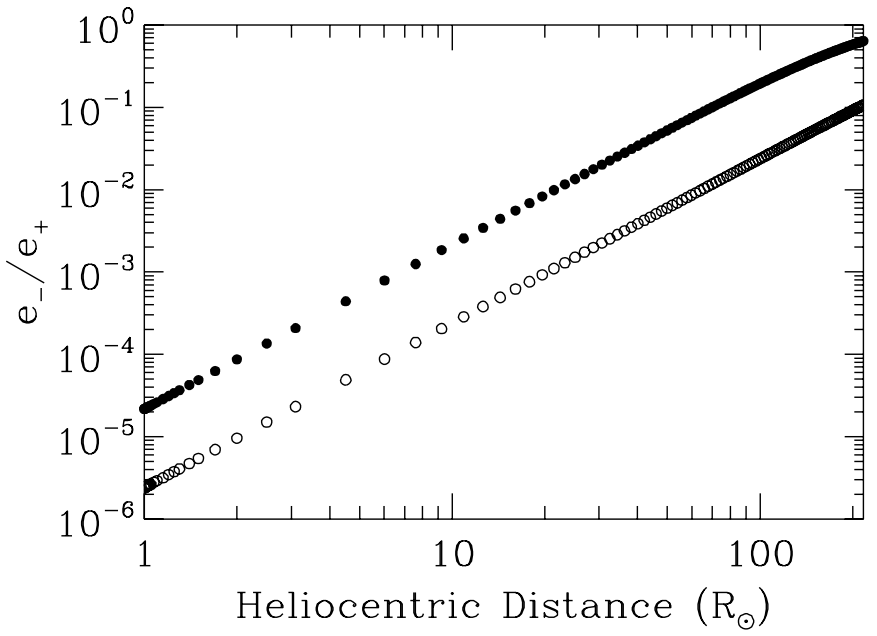

Fig. 9. The ratio of sunward to anti-sunward propagating wave power. Open circles: based on Eq. (5); filled circles: based on Eq. (26).

high as $650 \mathrm{~km} \mathrm{~s}^{-1}$ in the solution. Thus, the available wave power alone in these calculations seems to be able to drive the solar wind velocity to the high-speed range. Because of that, these solutions correspond to the case of a strong wave-driven solar wind.

As the ions in the two solar wind solutions have similar energies, outflow speeds, and temperatures, their velocity distributions are also similar. Figure 8 shows the cuts of the normalized proton velocity distributions at the $v_{\perp}=0$ plane of these strong wave-driven solutions. We can see that the distributions of the two solutions do not show a significant difference (open circles and crosses in Fig. 8). For these solutions, there are no clear indications of a secondary peak. We have generated a different strong wave-driven solution with kinetic suprathermal electron effects considered. The parameters of this solution are identical to the cases discussed above, except that the ratio of sunward to anti-sunward propagating wave power, instead of Eq. (5), is replaced by the following expression:

$e_{-} / e_{+}=1-\mathrm{e}^{-s^{2}}$.

As shown in Fig. 9, $e_{-} / e_{+}$is larger by about an order of magnitude with Eq. (26) replacing Eq. (5). The increase in the sunward propagating wave power does not seem to affect the core region of the proton velocity distributions by much, as indicated in Fig. 8. However, it has a stronger effect on the tail of the distributions. With the increase $e_{-} / e_{+}$ratio, indications of the formation of a secondary peak at the tail are much stronger (filled circles in Fig. 8). This result supports the association between double-peaked proton velocity distributions and sunward propagating waves that was discussed earlier.

Overall, it is clear from our comparison of the ion energies, outflow speeds, temperatures, and distributions that when strong wave-particle interactions are present, the velocity filtration effect plays an insignificant role in the driving and acceleration of the solar wind. Recently, Vocks \& Marsch (2001, 2002) have introduced a semi-kinetic model for the solar wind to describe the effects of strong ion cyclotron resonant heating. Like this study, their model describes the waveparticle interactions in the framework of quasilinear theory. 
However, the treatment of the particles is different in the two models. Their model is based on reduced ion distributions, massless electron fluid, and an assumed electron temperature profile. With the approximations on the electrons, the model does not take into account the kinetic effects associated with the suprathermal electrons. However, as we have shown that the velocity filtration effect associated with the suprathermal electrons is negligible when strong ion resonant heating is present, Vocks \& Marsch's assumptions regarding the electrons seem to be reasonable approximations.

\subsection{Weak wave-driven case}

Kinetic suprathermal electron effects were proposed to be a solar wind acceleration mechanism under the assumption that no wave-particle interactions were present (Olbert 1982). Therefore, it is worthwhile for us to investigate the velocity filtration effect in the scenario of a weak wave-driven solar wind. In this scenario, we assume that the available wave power for ion resonances is not sufficient to drive the outflow to the velocity range of the fast solar wind. We are interested in finding out whether the velocity filtration effect can make a considerable contribution in driving the solar wind in this situation.

Using a similar approach as in Sect. 3.1, we compare two solar wind solutions for the weak wave-driven case, one with the kinetic effects associated with the suprathermal electrons taken into account, and the other with a Maxwellian approximation for the entire electron distribution. These calculations essentially share the same boundary conditions as those in the previous section, but with two exceptions. First, $\eta_{0}$ is reduced to 0.0075 to characterize the weak wave-driven scenario. Second, as indicated in Fig. 5, the profiles for $\mathcal{E}$ have plateaued long before the distance reaches $1 \mathrm{AU}$. Therefore, for the present purpose, it is sufficient for us to set the upper boundary of the calculations to $0.1 \mathrm{AU}\left(\approx 21.5 R_{\odot}\right)$.

With the reduced level of available wave power, and without the suprathermal electron effects, we find that both the proton and helium ion speeds are only about $390 \mathrm{~km} \mathrm{~s}^{-1}$ at $0.1 \mathrm{AU}$ (Fig. 10). The inclusion of kinetic suprathermal electron effects

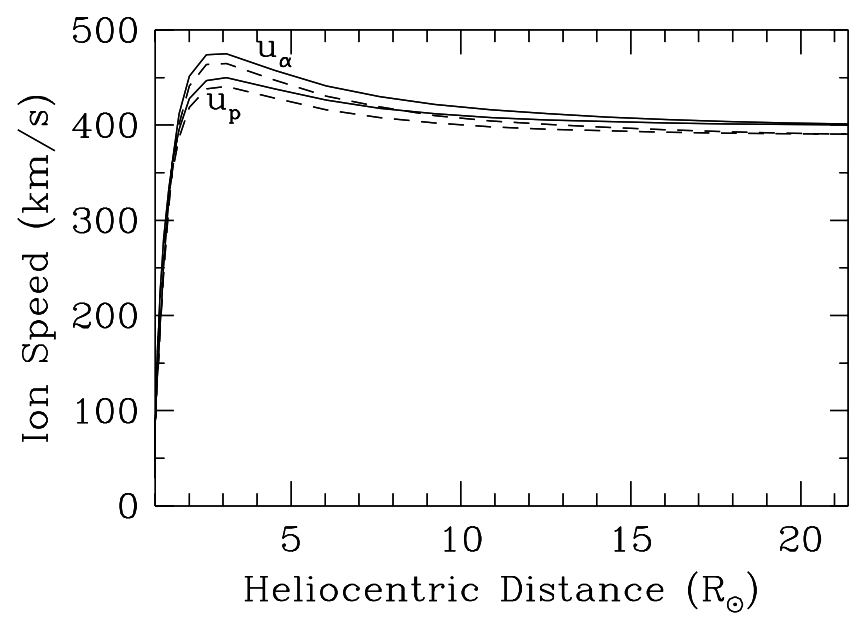

Fig. 10. Profiles of the proton and helium ion outflow speeds for the weak wave-driven solar wind case. Solid lines: kinetic suprathermal electron effects included; dashed lines: with a Maxwellian approximation for the entire electron distributions.

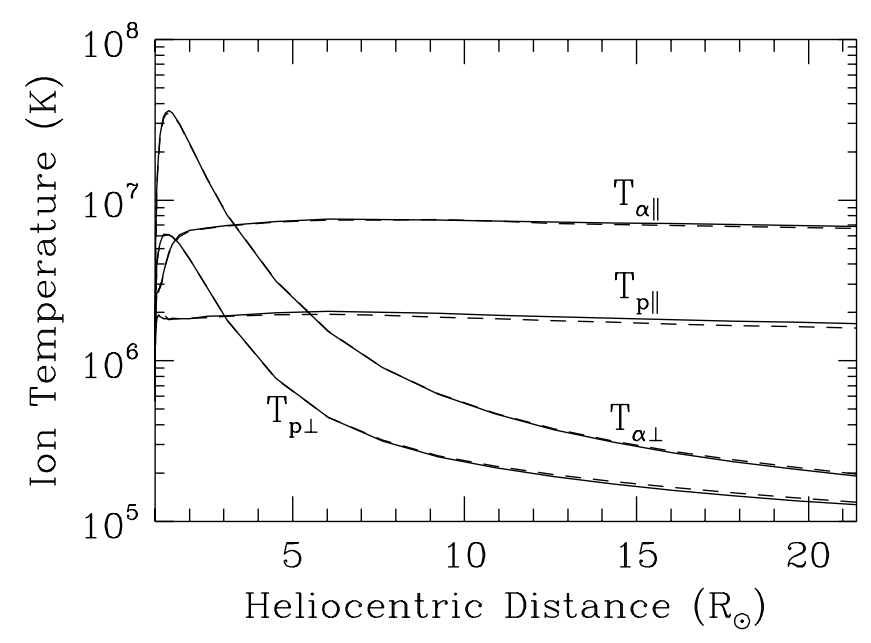

Fig. 11. Ion temperature profiles for the weak wave-driven solar wind case. Solid lines: kinetic suprathermal electron effects included; dashed lines: with a Maxwellian approximation for the entire electron distributions.

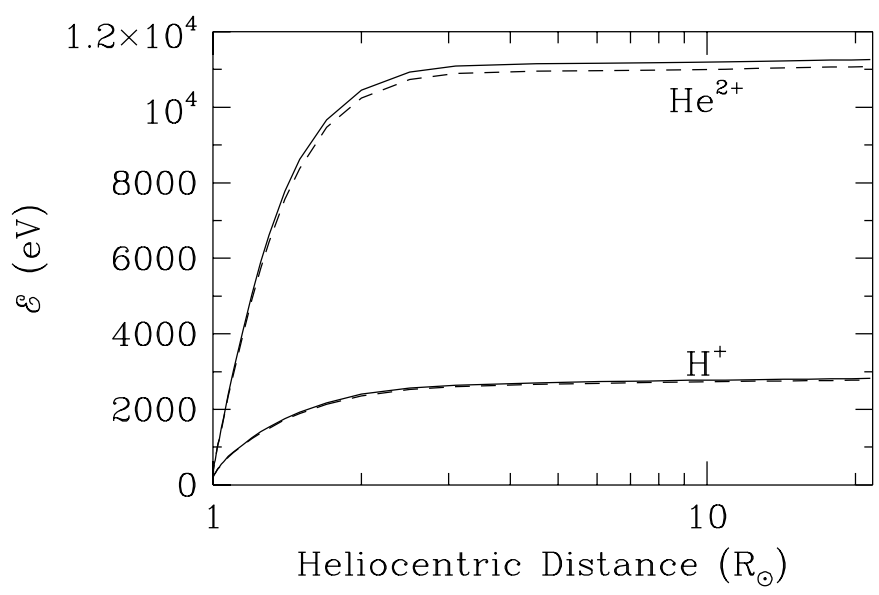

Fig. 12. $\mathcal{E}$ for protons and helium ions in the weak wave-driven solar wind scenario. Solid lines: kinetic suprathermal electron effects included; dashed lines: with a Maxwellian approximation for the entire electron distributions.

increases these speeds only by $2.5 \%$ for the protons and $2.8 \%$ for the helium ions.

With the reduced wave power, the ion parallel temperatures, as shown in Fig. 11, are smaller by about half compared with the corresponding values in the strong wave-driven solar wind case (Fig. 6). However, as in the previous case, the velocity filtration effect does not seem to affect the ion temperature in the present scenario.

As for the energy quantity $\mathcal{E}$, comparison between Figs. 5 and 12 indicates that the values for both ion species reduce by about one-thirds due to the weaker wave power. By comparing the two solutions in Fig. 12, one can see that with the reduced wave power, the velocity filtration effect is still only responsible for a small portion of the total energy input to the ions. For both ion species, inclusion of the kinetic effect leads to an increase of less than $2 \%$ in the total energy input from $1 R_{\odot}$ to $0.1 \mathrm{AU}$. Therefore, even in a weak wave-driven scenario, the 
velocity filtration effect is negligible in terms of driving and accelerating the solar wind.

\section{Discussion}

Our results have shown that ion cyclotron resonant heating is a much more efficient solar wind driving and acceleration mechanism compared with the velocity filtration effect that associates with the suprathermal electrons. Nevertheless, kinetic suprathermal electron effects, in general, may not always be negligible. In situations where there are physical processes which affect the suprathermal electron population more strongly than Coulomb collisions, kinetic suprathermal electron effects may contribute to the solar wind acceleration considerably. In fact, the strength of these kinetic effects depends on the electron distribution. We note that the suprathermal electrons considered in this study come from the tail portion of a Maxwellian distribution, whose temperature is $100 \mathrm{eV}$. Based on our experience, a higher electron temperature, due to the kinetic effects of the suprathermal electrons, would translate into a larger total electron heat flux. That would lead to a larger ambipolar electric field, and consequently, higher ion outflow speeds. Such a dependence between the electron temperature and the solar wind flow speed has been demonstrated by Maksimovic et al. (1997). But perhaps more importantly, an electron distribution that deviates from the Maxwellian by having an enhanced suprathermal tail may affect the solar wind speed even more strongly. Maksimovic et al. (1997) and Pierrard et al. (1999) studied the effects of electron Kappa distributions on the solar wind from a few $R_{\odot}$ to $1 \mathrm{AU}$. They showed that in cases where the electron distributions had a very enhanced suprathermal tail, the solar wind velocities could approach the observed high-speed range. Although no physical processes were related to the possible formation of such highly non-thermal electron distributions, these authors pointed out the association between a Kappa-like distribution near the corona and the observed electron distributions at $1 \mathrm{AU}$.

A physical mechanism that can significantly enhance the tail portion of the solar wind electron distributions, and increase the electron temperature and heat flux is wave-particle interactions. Chashei \& Fahr (2000) suggested that electron heating due to, for example, preexisting whistler waves in the solar wind, is required to produce the electron heat flux observed by the Ulysses spacecraft. More recently, one of our studies (Tam \& Chang 2001) investigated the effects of electron cyclotron resonant heating in the solar wind. We showed that the electron heating by the right-hand polarized waves near the corona could raise the electron heat flux and temperature by an order of magnitude, and lead to a much larger ambipolar electric field due to the associated kinetic effects. The enhanced electric field resulted in a $16 \%$ increase in the proton outflow speed at $1 \mathrm{AU}$ for the strong wave-driven case. Thus, even though ion resonant heating appears to be the dominant solar wind driving mechanism, the kinetic effects associated with the suprathermal electrons can sometimes make a considerable contribution to the acceleration of the solar wind.

\section{Conclusions}

In this study, we have compared the effects of two solar wind acceleration mechanisms: wave-particle interactions and the kinetic effects of the suprathermal electrons. Because the suprathermal electron population in our calculations corresponds to the tail portion of a Maxwellian distribution, it has been implicitly assumed that these suprathermal electrons have not undergone any heating, such as that involving electron cyclotron resonances. In addition, besides Coulomb collisions, we do not consider any physical process which may strongly affect the shape of the electron distributions. Therefore, the kinetic suprathermal electron effects that we consider are essentially the velocity filtration effect, which arises due to Coulomb collisions and the global kinetic nature of the solar wind flow. Our results have shown that in the presence of ion cyclotron resonant heating, the contribution by the velocity filtration effect in the driving and acceleration of the solar wind is relatively insignificant. However, when there are other physical processes, such as electron cyclotron resonances, that strongly affect the suprathermal electrons, kinetic suprathermal electron effects may no longer be negligible.

Acknowledgements. The authors would like to thank Horst Fichtner for his constructive comments during the evaluation of the manuscript. This work is partially supported by AFOSR, NASA, and NSF.

\section{References}

Axford, W. I. 1968, J. Geophys. Res., 73, 6855

Banaszkiewicz, M., Axford, W. I., \& McKenzie, J. F. 1998, A\&A, 337, 940

Banks, P. M., \& Holzer, T. E. 1968, J. Geophys. Res., 73, 6846

Bavassano, B., Dobrowolny, M., Mariani, F., \& Ness, N. F. 1982, J. Geophys. Res., 87, 3617

Belcher, J. W., \& Davis Jr. L. 1971, J. Geophys. Res., 76, 3534

Book, D. L. 1989, in A Physicist's Desk Reference, 2nd edn., ed. H. L. Anderson (New York: American Institute of Physics), 282

Chang, T. 2002, to appear in the proceedings of Solar Wind 10

Chashei, I. V., \& Fahr, H. J. 2000, A\&A, 363, 295

Cranmer, S. R. 2001, J. Geophys. Res., 106, 24937

Cranmer, S. R., Kohl, J. L., \& Noci, G. 1998, Space Sci. Rev., 85, 341

Cranmer, S. R., Kohl, J. L., Noci, G., et al. 1999a, ApJ, 511, 481

Cranmer, S. R., Field, G. B., \& Kohl, J. L. 1999b, ApJ, 518, 937

Czechowski, A., Ratkiewicz, R., McKenzie, J. F., \& Axford, W. I. 1998, A\&A, 335, 303

Denskat, K. U., \& Neubauer, F. M. 1982, J. Geophys. Res., 87, 2215

Dusenbery, P. B., \& Hollweg, J. V. 1981, J. Geophys. Res., 86, 153

Feldman, W. C., Barraclough, B. L., Phillips, J. L., \& Wang, Y.-M. 1996, A\&A, 316, 355

Fichtner, H., Sreenivasan, S. R., \& Vormbrock, N. 1996, J. Plasma Phys., 55, 95

Gary, S. P., Goldstein, B. E., \& Steinberg, J. T. 2001, J. Geophys. Res., 106,24955

Hollweg, J. V., \& Turner, J. M. 1978, J. Geophys. Res., 83, 97

Hu, Y. Q., \& Habbal, S. R. 1999, J. Geophys. Res., 104, 17045

Hu, Y. Q., Esser, R., \& Habbal, S. R. 1997, J. Geophys. Res., 102, 14661

Ichimaru, S. 1973, Basic Principles of Plasma Physics: A Statistical Approach (Reading, Massachusetts: W. A. Benjamin)

Isenberg, P. A. 1984, J. Geophys. Res., 89, 6613 
Isenberg, P. A., Lee, M. A., \& Hollweg, J. V. 2000, Sol. Phys., 193, 247

Kohl, J. L., Noci, G., Antonucci, E., et al. 1998, ApJ, 501, L127

Leer, E., Holzer, T. E., \& Shoub, E. C. 1992, J. Geophys. Res., 97, 8183

Li, X., Habbal, S. R., Hollweg, J. V., \& Esser, R. 1999, J. Geophys. Res., 104, 2521

Liewer, P. C., Velli, M., \& Goldstein, B. E. 2001, J. Geophys. Res., 1046, 29261

Maksimovic, M., Pierrard, V., \& Lemaire, J. F. 1997, A\&A, 324, 725

Marsch, E., Mühlhäuser, K.-H., Rosenbauer, H., Schwenn, R., \& Neubauer, F. M. 1982a, J. Geophys. Res., 87, 35

Marsch, E., Mühlhäuser, K.-H., Schwenn, R., et al. 1982b, J. Geophys. Res., 87, 52

Marsch, E., Goertz, C. K., \& Richter, K. 1982c, J. Geophys. Res., 87, 5030

Olbert, S. 1982, NASA Conf. Publ., 149

Pierrard, V., Maksimovic, M., \& Lemaire, J. F. 1999, J. Geophys. Res., 104,17021

Pilipp, W. G., Miggenrieder, H., Mühlhäuser, K.-H., Rosenbauer, H., \& Schwenn, R. 1990, J. Geophys. Res., 95, 6305
Retterer, J. M., Chang, T., \& Jasperse, J. R. 1983, Geophys. Res. Lett., 10,583

Schunk, R. W. 1977, Rev. Geophys. Space Phys., 15, 429

Scime, E. E., Bame, S. J., Feldman, W. C., Gary, S. P., \& Phillips, J. L. 1994, J. Geophys. Res., 99, 23401

Scudder, J. D., \& Olbert, S. 1979, J. Geophys. Res., 84, 2755

Steinberg, J. T., Lazarus, A. J., Ogilvie, K. W., Lepping, R., \& Byrnes, J. 1996, Geophys. Res. Lett., 23, 1183

Swanson, D. G. 1989, Plasma Waves (San Diego, CA: Academic Press)

Tam, S. W. Y., \& Chang, T. 1999a, Geophys. Res. Lett., 26, 3189

Tam, S. W. Y., \& Chang, T. 1999b, Comments on Modern Phys., 1, 141

Tam, S. W. Y., \& Chang, T. 2001, Geophys. Res. Lett., 28, 1351

Tam, S. W. Y., Yasseen, F., Chang, T., \& Ganguli, S. B. 1995, Geophys. Res. Lett., 22, 2107

Tam, S. W. Y., Yasseen, F., \& Chang, T. 1998, Ann. Geophys., 16, 948

Tu, C.-Y., \& Marsch, E. 1990, J. Geophys. Res., 95, 4337

Tu, C.-Y., \& Marsch, E. 2001, J. Geophys. Res., 106, 8233

Vocks, C., \& Marsch, E. 2001, Geophys. Res. Lett., 28, 1917

Vocks, C., \& Marsch, E 2002, ApJ, 568, 1030 